\title{
Internal Transitions of Charged Excitons in AlGaAs/GaAs Lateral Fluctuation Quantum Dots
}

\author{
C.J. Meining ${ }^{a}$, V.R. Whiteside ${ }^{a}$, B.D. $\mathrm{MCCombe}^{a}$, \\ H.A. Nickel ${ }^{a}$, A. Petrou ${ }^{a}$, J.G. Tischler ${ }^{b}$, A.S. Bracker ${ }^{b}$, \\ D. GAMmON ${ }^{b}$ AND A.B. DZYUBEnKo ${ }^{a, c}$ \\ ${ }^{a}$ Department of Physics and CAPEM, University at Buffalo \\ SUNY, Buffalo, NY 14260, USA \\ ${ }^{b}$ Naval Research Laboratory, Washington, D.C., 20375-5347, USA \\ ${ }^{c}$ Department of Physics, California State University Bakersfield \\ Bakersfield, CA 93311, USA
}

\begin{abstract}
Low temperature measurements of magneto-photoluminescence and optically detected resonance spectroscopy in magnetic fields up to $10 \mathrm{~T}$ were carried out on $\mathrm{GaAs} / \mathrm{Al}_{0.3} \mathrm{Ga}_{0.7} \mathrm{As}$ quantum well samples grown by molecular beam epitaxy with lateral fluctuation quantum dots produced by growth interruption. Monolayer fluctuations of the quantum well width form lateral quantum dots for which confinement energies are less than Coulomb correlation energies. Five different width quantum wells $(2.8-14.1 \mathrm{~nm})$ were grown in a single sample, doped in the barriers with $\mathrm{Si}$ donors to allow for photoluminescence of both excitons and trions. We report studies of the optically detected resonance spectra associated with the ensemble photoluminescence of all of the wells including observation of bound-to-continuum internal transitions of trions, both singlet and triplet, and electron cyclotron resonance for the wider wells, which also show a clear bound-to-bound triplet transition. The latter is forbidden by magnetic translational invariance, but can be seen in these samples because this symmetry is broken by the lateral fluctuations, whose characteristic dimensions are greater than the trion orbit size. The two narrowest wells show strong broad optically detected resonance signals associated with inhomogeneously broadened internal transitions of the strongly correlated trions in the lateral dots. The optically detected resonance signals peak well below the calculated positions of electron cyclotron resonance. As expected for localized carriers and excitons, there is no free electron cyclotron resonance. We also present preliminary measurements of optically detected resonance spectra from a single dot.
\end{abstract}

PACS numbers: 71.35.Ji, 71.35.Pq, 73.21.La, 78.55.Cr, 78.67.Hc 


\section{Introduction/background}

Over the past 10-15 years two classes of quantum dot structures derived from semiconductor heterostructures have been studied extensively by optical techniques: self-assembled quantum dots produced by the Stranski-Krastanow growth; and so-called interface fluctuation quantum dots [1-4] produced by interrupted growth at the heterointerfaces of narrow quantum wells. In the former case for structures such as $\operatorname{In}(\mathrm{Ga}) \mathrm{As} / \mathrm{GaAs}$ [5], the confinement energies in all three spatial directions typically exceed the electron-hole Coulomb correlation energy (exciton binding energy) of photoexcited electrons and holes by at least an order of magnitude. On the other hand, in the case of lateral fluctuation quantum dots strong confinement exists only along the growth direction, and the confinement in the lateral directions arises from monolayer differences in well width; in the AlGaAs/GaAs system this is typically a few meV, while the quasi-2D exciton binding energy is approximately $10 \mathrm{meV}$. This suggests rather different physical starting points for discussing the electronic energy states in these structures. Typically the electronic states of empty self-assembled dots are described by considering the single particle energy levels of the quantum dot "atom" for the conduction and valence bands and "turning on" the Coulomb correlation subsequently to determine the excitonic states. A more physically transparent starting point for a description of electronic states of a single electron-hole pair in a lateral fluctuation quantum dot with strong vertical confinement and weak lateral confinement (typical sizes of the order of $20-40 \mathrm{~nm}$ ) is a quasi-2D exciton confined laterally in a suitable $2 \mathrm{D}$ box with low barriers. The excited states of vertical confinement lie at much higher energies than any of the lateral confinement states, and the effective Bohr radius of the quasi-2D exciton is roughly $5 \mathrm{~nm}$, considerably less than the extent of the lateral confinement. Thus the lowest lying excited states are the quantized states of the center of mass (CM) exciton in the lateral confining box. All the internal excited states of the quasi-2D exciton lie at somewhat higher energies than the lowest few CM confinement states. This is the zeroth order picture; the internal degrees of freedom and the CM degrees of freedom are generally coupled, modifying this simple picture. It is of interest to explore this coupling and the details of the energy states of this highly correlated system by a suitable technique. It is of particular interest to determine the states of charged excitons in this system because of interesting electron-electron correlations and possible applications of charged excitons in quantum dots as Q-bits [6]. There have already been several previous investigations of both neutral and charged excitons in lateral fluctuation quantum dots by photoluminescence (PL), magneto-photoluminescence (MPL) and photoluminescence excitation (PLE) (e.g., [7, 8]).

The excited states of excitons and trions in $2 \mathrm{D}$ systems have received rather little experimental attention (see, e.g., [8-10]), and there has been even less work in quantum dots $[7,11,12]$. In addition, the coupling of the CM motion to the relative motion for interface fluctuation dots has received little experimental at- 
tention (see e.g. [7]). Theoretical predictions of the effect of lateral confinement on internal transitions of $\mathrm{X}$ and $\mathrm{X}^{-}$are available [13], and experimental work on internal transitions has been carried out via optically detected resonance (ODR) spectroscopy in quasi-2D systems. ODR spectroscopy is a sensitive technique in which sample PL is excited (typically) by a visible laser, and the small modulation of this characteristic PL induced by resonant absorption of a far infrared (terahertz) laser beam is monitored as a function of some external parameter, usually magnetic field $[9,10,14]$. This allows sensitive probing of electronic structure in the $\mathrm{THz}$ region (resonance is usually achieved by tuning the electronic states via an external magnetic field). Our present goal is to elucidate the nature of the confined states in lateral fluctuation quantum dots, and also to determine how the random distribution of weak lateral confining potentials in the case of the wider wells affects the internal transitions of the quasi-2D excitons. The data taken in a sample with a series of quantum wells with different quantum well widths and therefore different strengths of lateral fluctuating potential are compared with ODR data on a multiple quantum well sample with nominally smooth interfaces. These samples are modulation doped to study both excitons and negatively charged excitons.

\section{Samples and experiment}

Two samples grown by molecular beam epitaxy on semi-insulating GaAs (100) substrates have been studied: sample 1 - GaAs/ $\mathrm{Al}_{0.3} \mathrm{Ga}_{0.7} \mathrm{As}$ multiple-quantum-well (QW) structure containing 40 repetitions of $20 \mathrm{~nm}$ GaAs wells separated by $40 \mathrm{~nm}$ barriers doped in the barrier centers with $\mathrm{Si}$ at a sheet density of $2 \times 10^{10} \mathrm{~cm}^{-2}$ and symmetrized by a doping sheet of density $1 \times 10^{10} \mathrm{~cm}^{-2}$ at a distance of $20 \mathrm{~nm}$ from the bottom and top wells in the thick barriers; and sample $2-\mathrm{a} \mathrm{GaAs} / \mathrm{Al}_{0.3} \mathrm{Ga}_{0.7} \mathrm{As}$ structure containing five single QWs of nominal widths $2.8,4.2,6.2,8.4$, and $14.1 \mathrm{~nm}$ with the narrowest well on top. The QWs are separated by $40 \mathrm{~nm} \mathrm{Al}_{0.3} \mathrm{Ga}_{0.7}$ As barriers of which $3 \mathrm{~nm}, 10 \mathrm{~nm}$ from the top of each well, were silicon doped at a density of $10^{17} \mathrm{~cm}^{-3}$ (providing nominal sheet density of $3 \times 10^{10} \mathrm{~cm}^{-2}$ in each well). The growth was interrupted at the well/barrier interfaces to form the monolayer high lateral islands or dots [8]. A sample for the single dot experiments was fabricated from this wafer by depositing an $\mathrm{Al}$ mask on the surface and defining an array of indexed apertures with diameters between 25 microns and $200 \mathrm{~nm}$ by e-beam lithography. This approach has been described in detail elsewhere $[7,8]$.

The PL and ODR data for sample 1 were acquired with a 9 T superconducting magnet system with a variable temperature insert, a dual fiber optic coupling arrangement for excitation (with a He:Ne laser) and PL, an optically pumped far infrared (FIR) laser, light pipe optics for the FIR beam, a 3/4 m grating spectrometer and a photomultiplier tube (PMT), with a chopped FIR laser beam and lock-in detection. This system has been described previously [9]. 


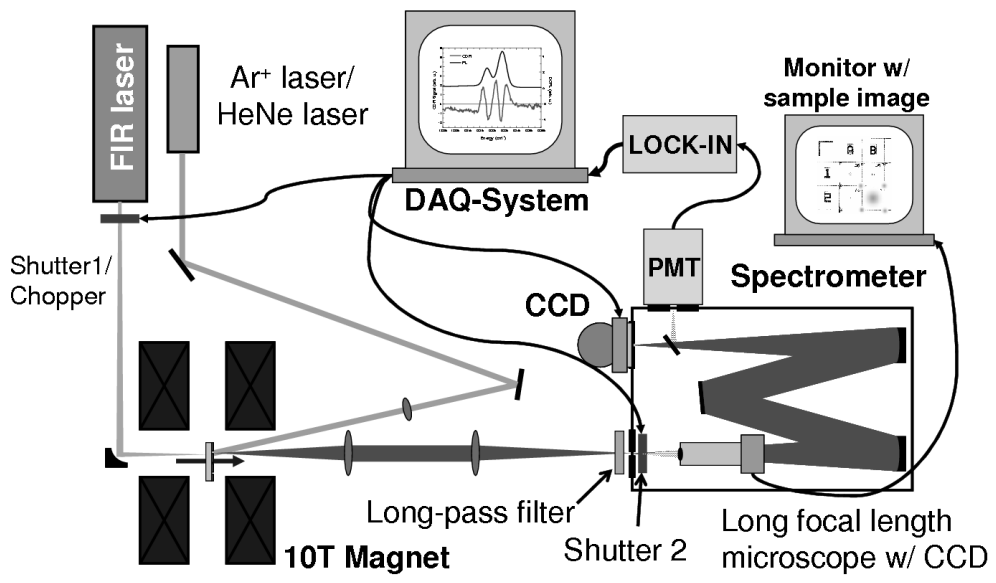

Fig. 1. Schematic diagram of the optical setup for studying single lateral fluctuation quantum dots via PL and ODR spectroscopy.

The data for the quantum dot sample (sample 2) were acquired with the optical/superconducting magnet system shown in Fig. 1. The sample is placed at low temperature in a variable temperature insert in a $10 \mathrm{~T}$ split-coil superconducting magnet with appropriate windows. The QD ensemble PL was excited with the $632.8 \mathrm{~nm}$ line of a He:Ne laser, and analyzed with a $3 / 4 \mathrm{~m}$ grating spectrometer equipped with a thermoelectrically cooled PMT and a liquid nitrogen cooled charged coupled device camera. The chopped output of an optically pumped molecular gas laser was used to modulate the PL at different FIR laser wavelengths. The spectral resolution for the slit width used for the ensemble spectra was about $0.5 \mathrm{meV}$; the grating position was controlled by computer to track the diamagnetic shift of a particular PL feature for the PMT data. The modulated output of the PMT is fed to a lock-in amplifier referenced to the frequency of the FIR chopper. This ODR signal, proportional to the difference between the PL signal with the FIR laser on and off, was recorded both as a function of PL energy and magnetic field. Some data were also acquired with the CCD system. In this case an optical shutter replaced the chopper, and a CCD "picture" of the spectrum was taken with the FIR laser beam on (for $4.5 \mathrm{~s}$ ) and a subsequent picture taken with the FIR laser beam off (for $4.5 \mathrm{~s}$ ) — the difference between the complete spectra with FIR laser on and off was then taken digitally and the result plotted appropriately. Photoluminescence for the apertured sample was obtained via a CCD camera/long focal length imaging microscope inside the spectrometer; the indexing of the sample made it possible to return easily to the same aperture for repeated measurements. The spectrometer resolution for the single dot spectra was about $0.25 \mathrm{meV}$. The two techniques were also compared in several cases for the ensemble spectra and gave good agreement. 


\section{Results}

\subsection{Magneto-photoluminescence}

Ensemble MPL spectra from all five wells in sample 2 are shown in Fig. 2 at $10 \mathrm{~T}$ and $4.2 \mathrm{~K}$. The multiple lines are a result of the well width fluctuations and recombination associated with both neutral $(\mathrm{X})$ and negatively charged $\left(\mathrm{X}^{-}\right)$ excitons. Features associated with the well-width fluctuations are not well resolved (at this resolution) for the widest well $(14.1 \mathrm{~nm}$ ) for which the difference in exciton energies for the two well widths is smaller than the $\mathrm{X}^{-}$binding energy. The difference in exciton energies for the monolayer width fluctuation for the narrowest well is much larger and dominates the $\mathrm{X}^{-}$binding energies, leading to a clearer picture. A more detailed view of the data from the widest and narrowest wells is given in Fig. 3, where the temperature dependence of the MPL features is shown

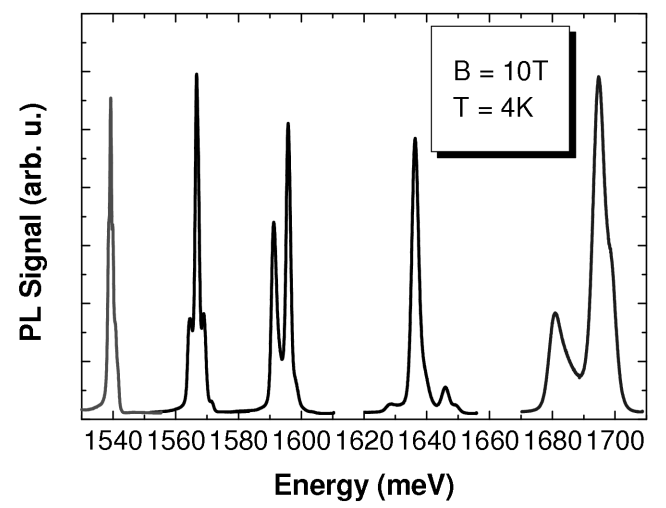

Fig. 2. PL spectra for all five well widths. From left to right the well widths are: $14.1 \mathrm{~nm}, 8.4 \mathrm{~nm}, 6.2 \mathrm{~nm}, 4.2 \mathrm{~nm}$, and $2.8 \mathrm{~nm}$.
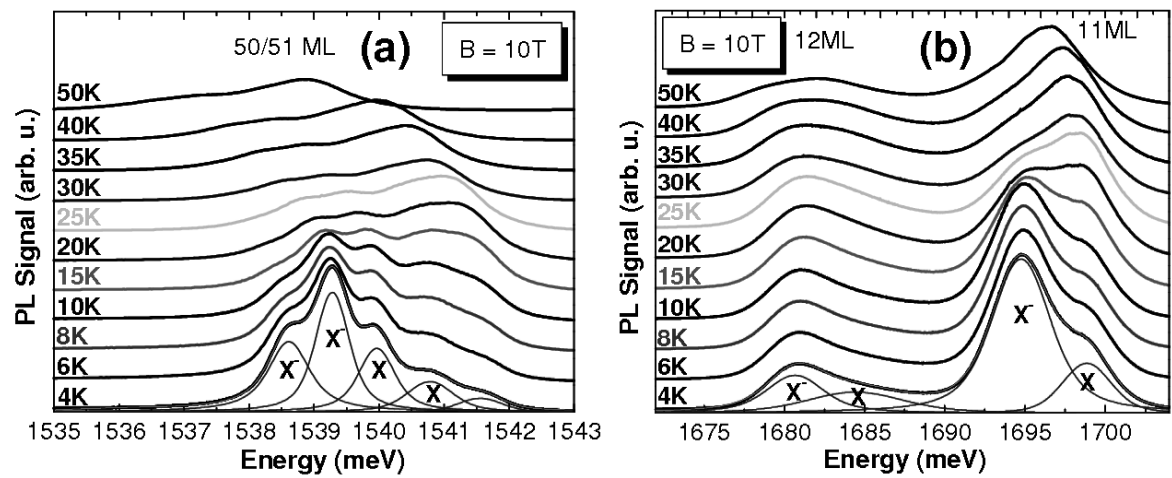

Fig. 3. Magneto-photoluminescence spectra for sample 2 at several temperatures: (a) for the $14.1 \mathrm{~nm}$ well width; (b) for the $2.8 \mathrm{~nm}$ well width. The individual curves labeled $\mathrm{X}_{\text {or } \mathrm{X}^{-}}$are the Voigt fits to the spectral features at $4.2 \mathrm{~K}$. 
along with the Voigt function fits to the $4.2 \mathrm{~K}$ data. The higher energy excitons are associated with the narrowest well widths. Calculations of the quantum well energy states, recent experiments [8] and calculations of the $\mathrm{X}^{-}$binding energies in the presence of lateral fluctuating potentials [15], and the temperature dependence shown in Figs. 3a and b, combined with the sign of the ODR signal for the different features permit assignment of the various observed lines (assignments are indicated on the fits to the $4.2 \mathrm{~K}$ data for each well width). Let us note that the $\mathrm{X}^{-}$binding energy reaches about $3.5 \mathrm{meV}$ for the narrowest well. At the highest temperatures studied, only the neutral excitons survive.

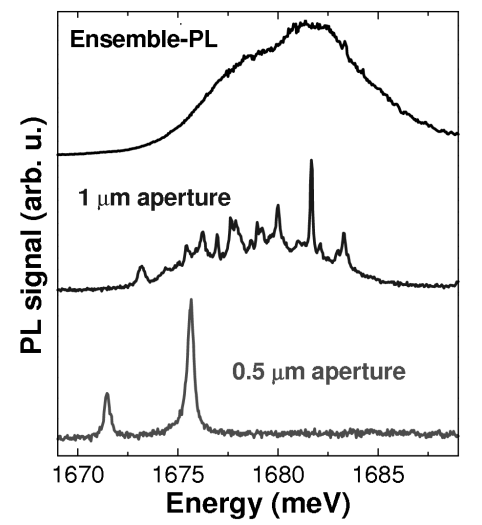

Fig. 4. PL spectra from lateral fluctuation Q-dots from a $2.8 \mathrm{~nm}$ wide, single GaAs $/ \mathrm{Al}_{0.3} \mathrm{Ga}_{0.7} \mathrm{As} \mathrm{Q}$-well. The spectra are obtained from a large area (top) through a 1 micron aperture (middle) and through a 0.5 micron aperture (bottom).

We have also observed and studied PL and MPL (not shown) from single dots for the narrowest well in sample $2(2.8 \mathrm{~nm})$. Figure 4 shows the evolution of the spectra from ensemble PL for a "large" lateral area (tens of microns ${ }^{2}$ ) which is inhomogeneously broadened due to contributions to the luminescence from many dots with varying dimensions, to the PL from a small aperture ( 0.5 micron diameter) which is limited to that of a single dot by further spatial resolution from limiting the vertical pixels of the CCD. The intermediate aperture shows an ensemble spectrum from a limited number of dots each with ultra-narrow lines with the width limited by the spectrometer resolution $(\approx 0.25 \mathrm{meV})$. Identification of individual features ( $\mathrm{X}$ or $\mathrm{X}^{-}$, from which dot) is difficult, and the size distribution of the dots has still not been extensively explored.

The ODR measurements for a single dot are very difficult, and we have focused initially on studying the ensemble spectra of the various wells with lateral fluctuations of varying magnitudes. We also present and discuss ODR spectra on a multiple-quantum-well sample with nominally smooth interfaces to provide a baseline for comparison. We have carried out some preliminary work on ODR of a single dot in the narrowest well, and this is also discussed briefly below. 


\subsection{Optically detected resonance spectroscopy}

Figure 5 shows ODR spectra for sample 1 at $4.2 \mathrm{~K}$ and a number of FIR laser lines. These data show electron cyclotron resonance (e-CR) as a very sharp line, and singlet and triplet internal transitions of negatively charged excitons (labeled $\mathrm{X}^{-} \mathrm{S}$ and $\mathrm{X}^{-} \mathrm{T}$ ), respectively. The internal transitions all correspond to excitations from a bound singlet or triplet state to a continuum with a sharp onset. More details of these allowed transitions can be found in Refs. [10] and [13]. Transitions from the ground state to bound excited states are forbidden by magnetic translational invariance [13]. The bound-to-bound triplet transition would occur close to but on the high field side of e-CR: there is no evidence of any feature in Fig. 5 in this field region for any of the laser lines at $4.5 \mathrm{~K}$ or at

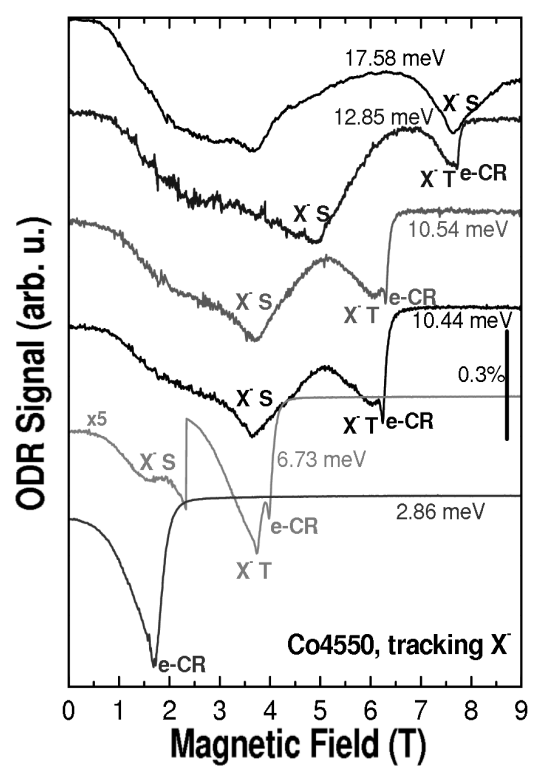

Fig. 5. ODR spectra from sample 1 (obtained by tracking the diamagnetic shift of the negatively charged exciton) at six FIR laser energies at $4.5 \mathrm{~K}$. The PL was excited by about $4 \mathrm{~mW}$ of $632.8 \mathrm{~nm}$ light from a He:Ne laser. The vertical bar indicates the size of the ODR signal as a percentage of the PL signal for the $10.44 \mathrm{meV}$ line.

any temperature up to $20 \mathrm{~K}$. We have also studied other samples carefully, and we have found no evidence of this bound-to-bound transition in the ODR signals in any sample with nominally smooth interfaces.

By contrast sample 2 shows a clear shoulder at fields above e-CR in the ensemble ODR data for the two widest wells (see Fig. 6, up arrows) at an FIR laser photon energy of $10.4 \mathrm{meV}$. The triangles indicate the calculated positions of free electron $\mathrm{CR}$ for each well including the effects of nonparabolicity in a simple two-band model $[16,17]$. We focus on the widest well sample to compare most 


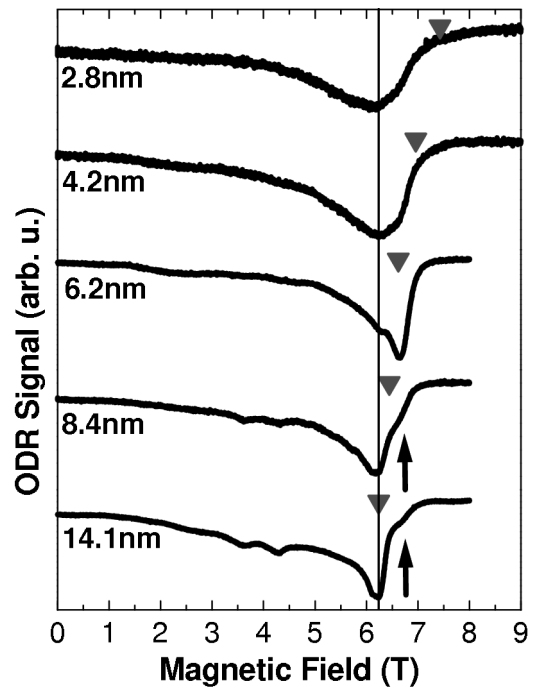

Fig. 6. Ensemble ODR spectra for all five wells of sample 2 at $4.2 \mathrm{~K}$ and a FIR laser photon energy of $10.4 \mathrm{meV}$. The triangles indicate the calculated position of free electron CR, accounting for the non-parabolicity due to confinement in the different wells. The up arrows indicate the position of the bound-to-bound transition discussed in the text.

directly with sample 1. Electron CR is the sharp feature at approximately $6.2 \mathrm{~T}$. Below e-CR the minima at $3.6 \mathrm{~T}$ and $4.5 \mathrm{~T}$ mark the onsets of two bound-to-continuum transitions of the $\mathrm{X}^{-}$-singlet ground state associated with the lowest Landau levels [13]. The sharp feature just below e-CR corresponds to one of the allowed $\mathrm{X}^{-}$-triplet transitions; it is an excited state and becomes more distinct when the temperature is raised slightly [18]. As stated above, the clear feature on the high-field side of e-CR has not been previously observed. For the mobile $\mathrm{X}^{-}$complex, the selection rules dictated by magnetic translational invariance [13] allow only the aforementioned bound-to-continuum transitions. In samples with intentional growth interrupts however, translational invariance is broken by well-width fluctuations. This leads to the observation of the bound-to-bound transition of the $\mathrm{X}^{-}$-triplet, which is predicted to occur on the high-field side of e-CR [13]. A model 2D calculation with a parabolic confining potential gives results for the energy position in reasonable agreement. This assignment is consistent with a temperature study; the shoulder disappears above about $10 \mathrm{~K}$ [8]. The additional thermal energy is apparently enough to cause the quasi- $2 \mathrm{D} \mathrm{X} \mathrm{X}^{-}$complex to become insensitive to the weak fluctuating potential.

The ensemble ODR spectra for the narrowest wells are less amenable to straightforward interpretation. As can be seen from Fig. 6, there is little or no ODR signal at the calculated position of free electron CR. (The calculation was done with a two-band model; so the triangles represent a lower limit in field for the resonance [19].) This is not surprising since free carrier CR should not be possible 
at low carrier temperatures for the narrowest wells because the lateral confining potential is of the order of $10 \mathrm{meV}$, much larger than $k T$. In this field region the observed ODR signal must be associated with internal electron transitions of the correlated electron-hole system in the random confining potentials. Let us note that the peak of the inhomogeneously broadened ODR signal lies well below the calculated position of e-CR and there is a tail to even lower magnetic fields with no apparent structure corresponding to singlet bound-continuum transitions as seen in the wide wells (in the 3.5-4.5 T region). There is structure on the high-field side of the broad ODR minimum but still below e-CR for the two narrowest wells, and the dominant feature in the $6.2 \mathrm{~nm}$ well lies at the same position as the shoulder attributed to the bound-bound triplet transition in the two widest wells.

We do not understand this interesting and complex behavior, at present, and there are as yet no detailed calculations for internal transitions of charged excitons in large, weakly confining lateral quantum dots to provide guidance. However, some insight into the ODR results for the narrowest wells can be gotten from simple considerations. We expect the "second electron" to be weakly bound in the $\mathrm{X}^{-}$complex, and thus a rough estimate of the transition energy (to a higher Landau level) in a weak potential can be obtained by considering this electron to be free in the dot, i.e., localized electron CR. We have carried out a simple calculation for a parabolic confining potential by adjusting the curvature such that the parabolic potential at the estimated radius of the dot has the energy of a monolayer barrier and accounting for the non-parabolicity enhanced effective mass. This calculation yields resonant fields near the peak in the ensemble ODR signal, which should represent contributions from a random array of dot sizes.

Figure 7 shows the ensemble ODR spectrum and single dot ODR spectra for the narrowest well. The single dot spectra were obtained with the CCD method,

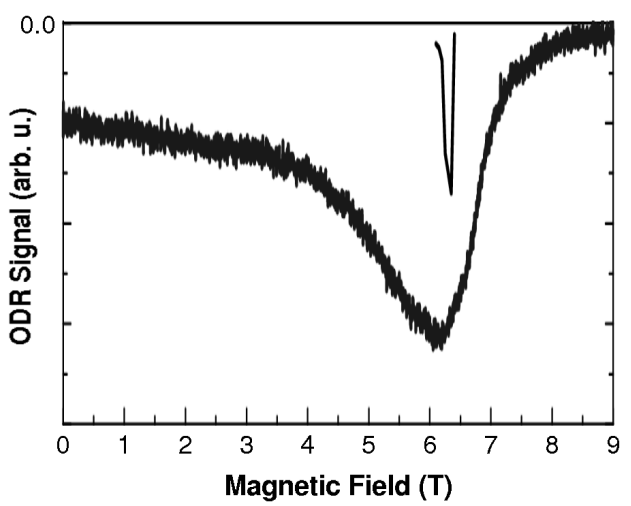

Fig. 7. Comparison of ensemble (broad grey curve) and single dot (sharp black line) ODR spectra for the narrowest well sample at $4.2 \mathrm{~K}$. Free electron CR would occur at $7.3 \mathrm{~T}$ or above accounting for non-parabolicity. Data were obtained for the $10.4 \mathrm{meV}$ line of the FIR laser with about $3 \mathrm{~mW}$ of He:Ne laser excitation at $632.8 \mathrm{~nm}$. 
and the plotted ODR lines represent the difference between the integrated intensity of the sharp multi-line MPL spectra with the FIR laser on and off. The negative-going signal is obtained by taking this difference for the $\mathrm{X}^{-}$lines, while the positive signal is obtained from the corresponding differences for the $\mathrm{X}$ features. We believe this is the first observation of internal transitions of a single dot. Let us note that the sharp single dot spectra occur near the peak of the ensemble ODR spectra, also consistent with the simple calculation outlined above.

\section{Summary}

We have studied the MPL and ODR spectra of two samples in fields up to $10 \mathrm{~T}$ at low temperature, a MQW structure with nominally smooth interfaces, and a sample containing 5 different wells with widths between 2.8 and $14.1 \mathrm{~nm}$, with growth interrupted interfaces to produce lateral fluctuation quantum dots. We have observed internal bound-to-bound triplet transitions in the widest wells of the sample with lateral potential fluctuations due to breaking of magnetic translational invariance. The narrowest wells show broad ODR signals with a peak significantly below the calculated position of free electron CR. We have also observed sharp ODR lines for a single dot in the narrowest well. The ODR signals are due to internal transitions of highly correlated negatively charged excitons weakly confined in the lateral $\operatorname{dot}(\mathrm{s})$. The details of the internal transitions are not yet understood, and much work, both experimental and theoretical, remains to be done.

\section{Acknowledgments}

This work was partially supported by NSF DMR 0203560 and NSF IGERT DGE0114330.

\section{References}

[1] D. Gammon, Al.L. Efros, J.G. Tischler, A.S. Bracker, V.L. Korenev, I.A. Merkulov, in: Quantum Coherence, Correlation and Decoherence in Semiconductor Nanostructures, Ed. T. Takagahara, Academic Press, London 2003, Ch. 6.

[2] A. Zrenner, L.V. Butov, M. Hagn, G. Abstreiter, G. Böhm, G. Weimann, Phys. Rev. Lett. 72, 3382 (1994).

[3] K. Brunner, G. Abstreiter, G. Böhm, G. Tränkle, G. Weimann, Phys. Rev. Lett. 73, 1138 (1994).

[4] H.F. Hess, E. Betzig, T.D. Harris, L.N. Pfeiffer, K.W. West, Science 264, 1740 (1994).

[5] M. Bayer, G. Ortner, O. Stern, A. Kuther, A.A. Gorbunov, A. Forchel, P. Hawrylak, S. Fafard, K. Hinzer, T.L. Reinecke, S.N. Walck, J.P. Reithmaier, F. Klopf, F. Schäfer, Phys. Rev. B 65, 195315 (2002) and references therein. 
[6] P. Chen, C. Piermarocchi, L.J. Sham, D. Gammon, D.G. Steel, Phys. Rev. B 69, 075320 (2004).

[7] D. Gammon, E.S. Snow, B.V. Shanabrook, D.S. Katzer, D. Park, Phys. Rev. Lett. 76, 3005 (1996); D. Gammon, E.S. Snow, D.S. Katzer, Appl. Phys. Lett. 67, 2391 (1995).

[8] J.G. Tischler, A.S. Bracker, D. Gammon, D. Park, Phys. Rev. B 66, 0813103 (2002).

[9] H.A. Nickel, G. Kioseoglou, T. Yeo, H.D. Cheong, A. Petrou, B.D. McCombe, D. Broido, K.K. Bajaj, R.A. Lewis, Phys. Rev. B 62, 2773 (2000).

[10] H.A. Nickel, T.M. Yeo, A.B. Dzyubenko, B.D. McCombe, A. Petrou, A.Yu. Sivachenko, W. Schaff, V. Umansky, Phys. Rev. Lett. 88, 056801 (2002).

[11] R.A. Child, R.J. Nicholas, N.J. Mason, P.A. Shields, J.-P.R. Wells, I.V. Bradley, J. Phillips, B.N. Murdin, Phys. Rev. B 68, 165307 (2003).

[12] B.N. Murdin, A.R. Hollingworth, J.A. Barker, D.G. Clarke, P.C. Findlay, C.R. Pidgeon, J.-P.R. Wells, I.V. Bradley, S. Malik, R. Murray, Phys. Rev. B 62, R7755 (2000).

[13] A.B. Dzyubenko, A.Yu. Sivachenko, Phys. Rev. Lett. 84, 4429 (2000).

[14] J. Kono, S.T. Lee, M.S. Salib, G.S. Herold, A. Petrou, B.D. McCombe, Phys. Rev. B 52, R8654 (1995) and references therein.

[15] A.V. Filinov, C. Riva, F.M. Peeters, Yu.E. Lozovik, M. Bonitz, arXiv:condmat/0401466, 2004.

[16] W. Zawadzki, J. Phys. C 16, 229 (1983).

[17] W. Seidenbusch, G. Lindemann, R. Lassnig, J. Edlinger, G. Gornik, Surf. Sci. 142, 375 (1984).

[18] C.J. Meining, V.R. Whiteside, B.D. McCombe, A. Petrou, J.G. Tischler, A.S. Bracker, D. Gammon, A.B. Dzyubenko, to be published.

[19] R.J. Warburton, J.G. Michels, R.J. Nicholas, J.J. Harris, C.T. Foxon, Phys. Rev. B 46, 13394 (1992). 